\title{
Introducing Arthritis Research \& Therapy's 10th anniversary issue, 'The Scientific Basis of Rheumatology: A Decade of Progress'
}

\author{
lain Hrynaszkiewicz ${ }^{1}$, Peter E Lipsky² and Ravinder N Maini² \\ ${ }^{1}$ Managing Editor, Arthritis Research \& Therapy, 34-42 Cleveland Street, London, W1T 4LB, UK \\ 2Editor-in-Chief, Arthritis Research \& Therapy, 34-42 Cleveland Street, London, W1T 4LB, UK
}

Published: 10 October 2008

This article is online at http://arthritis-research.com/content/10/5/119

(c) 2008 BioMed Central Ltd
Arthritis Research \& Therapy 2008, 10:119 (doi:10.1186/ar2522)

The first part of the special issue will include 9 articles. Subsequent parts will include the additional 31 articles that will complete the special issue. These will be available online immediately upon peer review and acceptance, and subsequently in printed issues of Arthritis Research \& Therapy. Immediate online publication is consistent with the commitment of Arthritis Research \& Therapy to provide rapid availability of important contributions to the field and also the capacity to search for relevant references in an integrated manner, whereas print publication gives the reader the advantages of a traditional review text.

\section{Overview of part one of The Scientific Basis of Rheumatology: A Decade of Progress Biology and methodology}

The contribution of Foxwell and colleagues [1] focuses on the role of signalling pathways in macrophages, the principal innate immune effector cells of rheumatoid arthritis. Evidence of the influence of innate immune cells in disease progression is also reviewed.

Mesenchymal stem cells (MSCs), nonhematopoietic progenitor cells found in various adult tissues, have the potential to repair tissue damage caused by osteoarthritis and rheumatoid arthritis. Chen and Tuan [2] discuss the potential involvement of MSCs in the pathophysiology of degenerative joint disease and explore the prospects of MSC-based therapies for these common rheumatic conditions.

Evidence for the involvement of epigenetic factors in rheumatic diseases has accumulated over several decades. However, in recent years, the effects of environmental triggers that cause heritable genetic changes without altering nucleotide sequence are becoming more widely elucidated, especially in the pathogenesis of rheumatoid arthritis. Gay and colleagues [3] explore the experimental evidence for the

$\mathrm{MSC}=$ mesenchymal stem cell. 


\section{Box 1. The growth of Arthritis Research \& Therapy and open access publishing}

All primary research articles have been freely available through the National Institutes of Health (NIH) repository of biomedical research articles, PubMed Central, since its launch in 2000. Arthritis Research \& Therapy transferred to BioMed Central, the first open access publisher, the same year.

By being open access, all Arthritis Research \& Therapy research articles are freely and universally accessible online, making them much more visible compared with journals that publish all of their content behind subscription barriers. As of this writing, more than 90,000 articles, on average, are downloaded per month from the journal website.

Many publishers now offer open access options, as recognition of the model has grown. Many funding agencies now require deposition of accepted articles in a public repository such as PubMed Central. The Wellcome Trust and the Arthritis Research Campaign have established such policies, and more recently the NIH Public Access Policy came into effect in April 2008.

Arthritis Research \& Therapy has now published over 700 original research articles and more than 200 reviews. The journal received its first Impact Factor in 2003, placing it third in the field. Other measures of a journal's success such as the SClmago Journal Rank also rank the journal highly.

role of epigenetic risk factors, which may assist in the development of novel molecular-based therapy.

Szekanecz and Koch [4] review the crucial involvement of the vasculature in inflammatory rheumatic diseases, including the role of the vasculature in atherosclerosis and increased cardiovascular risk, leading to increased mortality. The cellular mechanisms underlying these pathologic processes are described, and the possibility of therapeutic vascular targeting summarized.

Immunoablative therapy and hematopoietic stem cell transplantation in autoimmune diseases are the subjects of the article by Hügle and van Laar [5]. They review the available evidence of this intensive therapy - traditionally, a salvage therapy for nonresponders to conventional treatments - from retrospective cohort analyses, case series and translational studies and look forward to the outcomes of prospective randomized controlled clinical trials in systemic sclerosis and systemic lupus erythematosus.
Our view of rheumatic diseases has also changed substantially with the development of imaging techniques, which have improved our ability to observe the structure and function and pathogenic mechanisms of rheumatic disease more effectively. As discussed by McGonagle and Tan [6], magnetic resonance imaging permits the in vivo imaging of pathogeneic mechanisms in rheumatoid arthritis, with future technology potentially being able to explore disease at the cellular and molecular levels.

\section{Specific rheumatic diseases}

Developments in the scientific understanding of systemic lupus erythematosus are the focus of the contribution of Ardoin and Pisetsky [7], who review recent research into immune abnormalities in the disease, the scientific basis of which is leading to opportunities for developing more targeted treatment and a 'new era' of clinical trials.

Gout, an increasingly common form of inflammatory arthritis (especially in the elderly), initiated through the deposition of

\section{Box 2. Benefits of publishing with Arthritis Research \& Therapy}

Online functionality enables rapid peer review and publication. Authors can expect to receive a first decision on their manuscript within six weeks of submission.

Research is published as a 'provisional PDF' immediately upon formal acceptance and is indexed in PubMed within 48 hours.

Authors can be published within as little as 2 to 3 months of initial submission.

Research articles have no length restrictions or limitations on the number of colour figures, tables, or supplementary materials that can be included, enabling scientists to produce a true record of their work. 


\section{The Scientific Basis of Rheumatology: years A Decade of Progress}

This is part of a special collection of reviews, The Scientific Basis of Rheumatology: A Decade of Progress, published to mark Arthritis Research \& Therapy's 10th anniversary.

Other articles in this series can be found at: http://arthritis-research.com/sbr

monosodium urate crystals in the joint, is explored in the article by So [8]. So reviews both recent scientific and clinical experiments, which have provided new insights into uric acid transport, the basis of inflammation in gout and novel hyperuricemia-reducing drug trials.

The past decade has also seen scientific and clinical developments within the idiopathic inflammatory myopathies, diseases with not-yet-determined pathogeneses. Lundberg and Grundtman [9] describe available evidence for the immunological and nonimmunological basis of disease and the implications for future treatment options.

The Scientific Basis of Rheumatology: A Decade of Progress should provide the reader with an easily accessible, comprehensive and user-friendly review of the remarkable progress in rheumatology in the past decade. We hope it will not only give the basic investigator a comprehensive overview of the state of knowledge of rheumatology, but also provide the clinician an opportunity to understand the remarkable scientific developments in rheumatology which serve as the basis for modern therapeutic programs.

\section{References}

1. Drexler SK, Kong PL, Wales J, Foxwell BM: Cell signalling in macrophages, the principal innate immune effector cells of rheumatoid arthritis. Arthritis Res Ther 2008, 10:216.

2. Chen FH, Tuan RS: Mesenchymal stem cells in arthritic diseases. Arthritis Res Ther 2008, 10:223.

3. Strietholt S, Maurer B, Peters MA, Pap T, Gay S: Epigenetic modifications in rheumatoid arthritis. Arthritis Res Ther 2008, 10:219.

4. Szekanecz Z, Koch AE: Vascular involvement in rheumatic diseases: 'vascular rheumatology'. Arthritis Res Ther 2008, 10:224.

5. Hügle T, van Laar JM: Stem cell transplantation for rheumatic autoimmune diseases. Arthritis Res Ther 2008, 10:217.

6. McGonagle $D$, Tan $A L$ : What magnetic resonance imaging has told us about the pathogenesis of rheumatoid arthritis - the first 50 years. Arthritis Res Ther 2008, 10:222.

7. Ardoin S, Pisetsky DS: Developments in the scientific understanding of lupus. Arthritis Res Ther 2008, 10:218.

8. So A: Developments in the scientific and clinical understanding of gout. Arthritis Res Ther 2008, 10:221.

9. Lundberg IE, Grundtman C: Developments in the scientific and clinical understanding of inflammatory myopathies. Arthritis Res Ther 2008, 10:220. 